\title{
Decay of a bound muon into a bound electron
}

\author{
M. Jamil Aslam®, ${ }^{1,2}$ Andrzej Czarnecki®, ${ }^{1}$ Guangpeng Zhang $\odot,{ }^{1}$ and Anna Morozova ${ }^{1}$ \\ ${ }^{1}$ Department of Physics, University of Alberta, Edmonton, Alberta T6G 2E1, Canada \\ ${ }^{2}$ Department of Physics, Quaid-i-Azam University, Islamabad 44000, Pakistan
}

(Received 14 May 2020; accepted 23 September 2020; published 13 October 2020)

\begin{abstract}
When a muon bound in an atom decays, there is a small probability that the daughter electron remains bound. That probability is evaluated. Surprisingly, a significant part of the rate is contributed by the negative energy component of the wave function, neglected in a previous study. A simple integral representation of the rate is presented. In the limit of close muon and electron masses, an analytic formula is derived.
\end{abstract}

DOI: 10.1103/PhysRevD.102.073001

\section{INTRODUCTION}

Electrostatic binding of a muon $\mu^{-}$in an atom changes its decay characteristics. Coulomb attraction decreases the phase space available to the decay products but enhances the daughter electron wave function. Muon motion smears the energy spectrum of electrons. All these effects largely cancel in the lifetime of the muon [1] but they do slow down the decay by a factor that, for small atomic numbers $Z$, reads

$\Gamma\left(\left(Z \mu^{-}\right) \rightarrow e \nu_{\mu} \bar{\nu}_{e} N\right)=\left(1-\frac{(Z \alpha)^{2}}{2}\right) \Gamma\left(\mu \rightarrow e \nu_{\mu} \bar{\nu}_{e}\right)$,

and can be interpreted as the time dilation; the characteristic velocity of the bound muon is $Z \alpha$.

Another possible effect, of primary interest in this paper, is the decay into an electron that remains bound to the nucleus $N$. For the actual small ratio of electron to muon masses, $m_{e} / m_{\mu} \simeq 1 / 207$, that process is very rare, especially for weak binding in atoms with moderate $Z$. We study it as a part of a program of characterizing bound muon decays, motivated by upcoming experiments COMET [2] and Mu2e [3].

Throughout this paper we use $c=\hbar=1$ and treat the nucleus $N$ as static, spin 0 , and pointlike, neglecting effects of its recoil and finite size. We denote its number of protons by $Z$. The notation $\left(Z \mu^{-}\right)$or $\left(Z e^{-}\right)$denotes a muon or an electron bound in the $1 \mathrm{~s}$ state, forming a hydrogen-like atom. We assume that no other particles are bound to the

Published by the American Physical Society under the terms of the Creative Commons Attribution 4.0 International license. Further distribution of this work must maintain attribution to the author(s) and the published article's title, journal citation, and DOI. Funded by SCOAP . nucleus (we neglect screening or Pauli blocking due to other electrons).

The decay $\left(Z \mu^{-}\right) \rightarrow\left(Z e^{-}\right) \nu_{\mu} \bar{\nu}_{e}$ was previously studied in the very elegant and detailed paper [4]. We reevaluate it and find discrepancies with that pioneering study, particularly for large values of $Z$. This is most likely explained by negative energy components of the Dirac wave functions, neglected in [4] (as discussed in its Appendix A). Here we use exact Dirac wave functions in the Coulomb field of a pointlike nucleus. Effects of extended nuclear charge distribution were found to be very small in [4] so we neglect them.

Earlier studies of the differences between the decay of a free and of a bound muon include [5-8]. More recently, the spectrum of produced electrons was determined in [9-11].

This paper is organized as follows. In Sec. II, momentum space wave functions are used to compute the rate $\Gamma\left[\left(Z \mu^{-}\right) \rightarrow\left(Z e^{-}\right) \nu_{\mu} \bar{\nu}_{e}\right]$, as in Ref. [4]. Significant differences are found so the result is checked with position space wave functions in Sec. III. That approach turns out to be much simpler; a one-dimensional integral representation is found, replacing the triple integral of Ref. [4]. In the limit of nearly equal masses, $m_{e} \rightarrow m_{\mu}$, the remaining integration is done analytically and a closed formula for the rate is obtained in Sec. IV. Section V presents conclusions and the Appendix summarizes the formalism of Ref. [4].

In order to deal with binding effects, we describe the initial and the final states by solutions of a stationary Dirac equation. We treat the weak interaction that leads to the decay as a harmonic perturbation. This description is exact to all orders in $Z \alpha$, including relativistic and thus positron and antimuon effects. We expect corrections to the result to be suppressed by powers of the fine structure constant $\mathcal{O}\left(\alpha(Z \alpha)^{n}\right), n>0$, due to real and virtual radiative effects. Additional corrections due to the finite mass of the nucleus and its structure are also expected. 
This theoretical framework has been used for other atomic processes such as electromagnetic decays of excited atomic states and interactions of an atom with an external field [12-18].

Our work is somewhat analogous to the first study of the bound electron magnetic moment, by Gregory Breit [12], who determined the gyromagnetic ratio $g=2\left[1+2 \sqrt{1-(Z \alpha)^{2}}\right] / 3$. In his study, a bound electron interacts with an external field, just like in the decay process considered here. In the limit $Z \rightarrow 0$, the $g$-factor tends to the free-electron value, $g \rightarrow 2$. Breit's result is exact to all orders in $Z \alpha$, just like we claim our result is.

Breit's 1928 calculation was analyzed from the point of view of a nonrelativistic effective theory [19]. That analysis reveals negative-energy contributions [see Fig. 2(b) and the discussion following Eq. (12) in Ref. [19]]. We conclude that the negative-energy contributions are correctly included in a treatment based on the Dirac wave function.

Our present study is conceptually parallel to Breit's. The only differences are that the initial and final states have different masses; and, instead of an external magnetic field, the electroweak field induces the transition. In both cases the full result is given by a double series, in powers of $Z \alpha$ (describing the binding) and of $\alpha$ (describing selfinteractions and, in case of the muon decay, real photon radiation). The structure of this double series is shown, for example, in Eq. (5) in Ref. [20]. We expect a similar series to exist for the present problem of the bound muon decay. The soundness of the underlying theory has been tested by experiments providing the best determination of the electron mass [21].

\section{MOMENTUM SPACE DERIVATION OF THE DECAY RATE}

\section{A. Wave function and its normalization in momentum space}

We consider the muon in the ground state of a hydrogen-like ion and are interested in the final-state electron also in the ground state. Both muon and electron wave functions are 1s solutions of the Dirac equation and differ only by the mass, respectively $m_{\mu}$ and $m_{e}$. Below we present formulas for a generic mass $m$. The position space wave function $\Phi(\boldsymbol{x})$ will be presented below in Eq. (18). Taking its Fourier transform (see Appendix 1 in Ref. [4]) we obtain

$$
\begin{aligned}
\tilde{\Phi}_{ \pm}(\boldsymbol{k}) & =\int \mathrm{d}^{3} x \Phi(\boldsymbol{x}) e^{-i \boldsymbol{k} \cdot \boldsymbol{x}} \\
& =\left(\begin{array}{c}
\tilde{f}(k) \phi_{ \pm} \\
\tilde{g}(k) \frac{\sigma \cdot \boldsymbol{k}}{k} \phi_{ \pm}
\end{array}\right) \quad k=|\boldsymbol{k}|,
\end{aligned}
$$

where $\phi_{+}=\left(\begin{array}{l}1 \\ 0\end{array}\right)$ and $\phi_{-}=\left(\begin{array}{l}0 \\ 1\end{array}\right)$ are two-component spinors describing spin projections $\pm 1 / 2$ on the $z$ axis. We assume that the muon decays in the state $\phi_{+}$. We will use the simplified notation $f, g=\tilde{f}(k), \tilde{g}(k)$ and the dimensionless variable $q=\frac{k}{m \alpha_{Z}}$,

$$
\begin{aligned}
f= & \frac{2^{\gamma+1} \Gamma(\gamma+1)}{q\left(m \alpha_{Z}\right)^{3 / 2}} \sqrt{\frac{\pi(1+\gamma)}{\Gamma(1+2 \gamma)}} \operatorname{Im}(1-i q)^{-\gamma-1}, \\
g= & \frac{2^{\gamma+1}(1-\gamma) \Gamma(\gamma)}{\alpha_{Z} q^{2}\left(m \alpha_{Z}\right)^{3 / 2}} \sqrt{\frac{\pi(1+\gamma)}{\Gamma(1+2 \gamma)}} \\
& \times \operatorname{Im}\left\{[1-i q(\gamma+1)](1-i q)^{-\gamma-1}\right\},
\end{aligned}
$$

where $\alpha_{Z}=Z \alpha, \gamma=\sqrt{1-\alpha_{Z}^{2}}$, and $\alpha \simeq 1 / 137$ is the fine structure constant. Equations (4) and (5) are numerically identical with Eqs. (A3) and (A4) in [4]; the functional form we present seems to lead to slightly faster numerical integrations. We employ the basis [[22], Eq. (3.7)],

$$
\begin{gathered}
w^{1}(\boldsymbol{k})=c\left(\begin{array}{c}
1 \\
0 \\
\frac{k_{z}}{k^{0}+m} \\
\frac{k+}{k^{0}+m}
\end{array}\right), \quad w^{2}(\boldsymbol{k})=c\left(\begin{array}{c}
0 \\
1 \\
\frac{k_{-}}{k^{0}+m} \\
-\frac{k_{z}}{k^{0}+m}
\end{array}\right), \\
w^{3}(\boldsymbol{k})=c\left(\begin{array}{c}
\frac{k_{z}}{k^{0}+m} \\
\frac{k_{+}}{k^{0}+m} \\
1 \\
0
\end{array}\right), \quad w^{4}(\boldsymbol{k})=c\left(\begin{array}{c}
\frac{k_{-}}{k^{0}+m} \\
-\frac{k_{z}}{k^{0}+m} \\
0 \\
1
\end{array}\right),
\end{gathered}
$$

with $c=\sqrt{\frac{k^{0}+m}{2 m}}, k^{0}=\sqrt{m^{2}+\boldsymbol{k}^{2}}$, and $k_{ \pm}=k_{x} \pm i k_{y}$. In analogy with Eq. (A6) in [4], we expand the bound wave function in this basis,

$$
\begin{aligned}
& \Phi_{+}(\boldsymbol{k})=\sqrt{\frac{2 m}{2 k^{0}}}\left[A_{+} w^{1}(\boldsymbol{k})+A_{-} w^{2}(\boldsymbol{k})\right. \\
& \left.+B_{+}^{\star} w^{4}(-\boldsymbol{k})+B_{-}^{\star} w^{3}(-\boldsymbol{k})\right] \\
& =\sqrt{\frac{k^{0}+m}{2 k^{0}}}\left[A_{+}\left(\begin{array}{c}
1 \\
0 \\
\frac{k_{z}}{k^{0}+m} \\
\frac{k_{+}}{k^{0}+m}
\end{array}\right)+A_{-}\left(\begin{array}{c}
0 \\
1 \\
\frac{k_{-}}{k^{0}+m} \\
-\frac{k_{z}}{k^{0}+m}
\end{array}\right)\right. \\
& \left.+B_{+}^{\star}\left(\begin{array}{c}
-\frac{k_{-}}{k^{0}+m} \\
\frac{k_{z}}{k^{0}+m} \\
0 \\
1
\end{array}\right)+B_{-}^{\star}\left(\begin{array}{c}
-\frac{k_{z}}{k^{0}+m} \\
-\frac{k_{+}}{k^{0}+m} \\
1 \\
0
\end{array}\right)\right] \text {. }
\end{aligned}
$$


For example, for the spin projection $+1 / 2$,

$$
\begin{gathered}
A_{+}=\sqrt{\frac{k^{0}+m}{2 k^{0}}}\left(f+\frac{k g}{k^{0}+m}\right), \\
A_{-}=0, \\
B_{+}^{\star}=\sqrt{\frac{k^{0}+m}{2 k^{0}}} k_{+}\left(-\frac{f}{k^{0}+m}+\frac{g}{k}\right), \\
B_{-}^{\star}=\sqrt{\frac{k^{0}+m}{2 k^{0}}} k_{z}\left(-\frac{f}{k^{0}+m}+\frac{g}{k}\right),
\end{gathered}
$$

in agreement with (A7) in [4] except for $B_{-}^{\star}$, for which we find the opposite overall sign. We proceed to check the normalization,

$$
\int \frac{\mathrm{d}^{3} k}{(2 \pi)^{3}}\left(\left|A_{+}\right|^{2}+\left|A_{-}\right|^{2}+\left|B_{+}\right|^{2}+\left|B_{-}\right|^{2}\right)=1 .
$$

We confirm that the $B_{ \pm}$part of this integral is very small, $0.16 \%$ even for $Z=80$, in agreement with a comment below (A8) in [4]. Indeed, the $B_{ \pm}$part of the normalization integral, interpreted as the probability of finding a positron in the atom, is $\mathcal{O}\left(\alpha_{Z}^{5}\right)$ when $Z \rightarrow 0$. For this reason, the negative energy components of the wave function were neglected in [4]. The positive and negative energy components can be separated by acting on the wave function with Casimir projectors,

$$
\begin{gathered}
P_{A}=\frac{m}{k^{0}}\left(w_{1} w_{1}^{\dagger}+w_{2} w_{2}^{\dagger}\right)=\frac{\not k+m}{2 k^{0}} \gamma^{0}, \\
P_{B}=\gamma^{0} \frac{\not k-m}{2 k^{0}},
\end{gathered}
$$

such that $P_{A, B}^{2}=P_{A, B}$ and $P_{A}+P_{B}=1$. We find that the rate calculated with $P_{A}$-projected wave functions is substantially larger than when full wave functions are used. These results are compared in Fig. 1, where we plot the rate divided by

$$
\Gamma_{0}=\frac{G_{\mathrm{F}}^{2} m_{\mu}^{5}}{192 \pi^{3}}
$$

the free muon decay rate at tree level, in the limit of a massless electron; $G_{\mathrm{F}}=\frac{\sqrt{2} g^{2}}{8 M_{W}^{2}}$ is the Fermi constant [23]. The solid line in Fig. 1 shows the full wave function result, and the dots show the result with projectors $P_{A}$. For small and moderate $Z$, up to $Z \simeq 40$, the results are close, and start to diverge quite strongly for larger nuclei.

Since the $B_{ \pm}$contribution to the normalization is small even for large $Z$, these results are unexpected. We thus

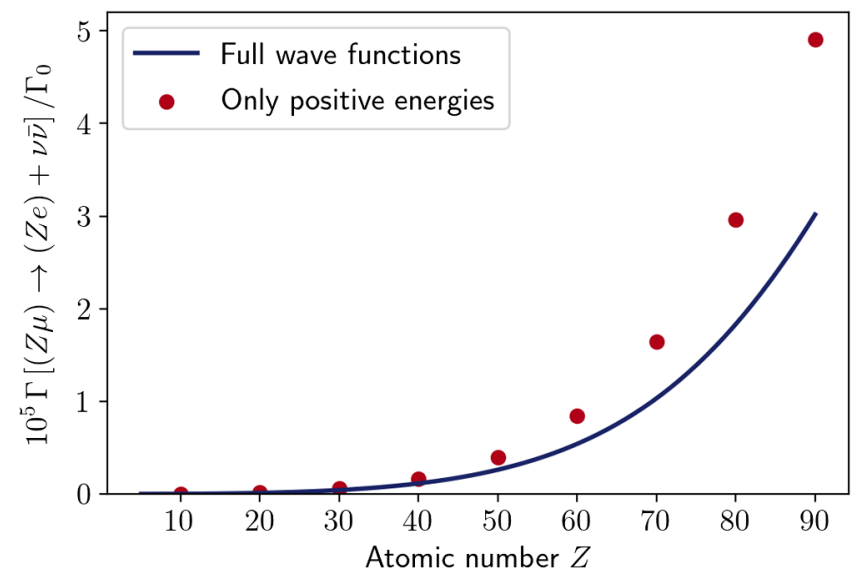

FIG. 1. Rate of the bound-to-bound decay $\left(Z \mu^{-}\right) \rightarrow\left(Z e^{-}\right)+$ $\nu \bar{\nu}$ normalized to the free muon decay rate $\Gamma_{0}$, as a function of the atomic number $Z$. The solid line shows our results found using complete wave functions. Red dots are the values obtained by neglecting negative energy components of wave functions. Errors due to this approximation grow with the atomic number $Z$.

proceed to check them in the position space. As a reward, we find that alternative method to be simpler. It will allow us to derive a closed formula for the rate in the limit of close electron and muon masses.

\section{BOUND $\mu^{-}$TO BOUND $e^{-}$DECAY IN POSITION SPACE}

In this section, we calculate the bound state transition rate $\left(Z \mu^{-}\right) \rightarrow\left(Z e^{-}\right) \nu_{\mu} \bar{\nu}_{e}$ using position space wave functions for the decaying muon and the produced electron [24],

$$
\Phi(\boldsymbol{r})=\psi_{n=1, j=\frac{1}{2}, \pm}(r, \theta, \phi)=\frac{f(r)}{\sqrt{4 \pi}} u_{ \pm},
$$

where

$f(r)=\left(2 m \alpha_{Z}\right)^{3 / 2} \sqrt{\frac{1+\gamma}{2 \Gamma(1+2 \gamma)}}\left(2 m r \alpha_{Z}\right)^{\gamma-1} \exp \left(-m r \alpha_{Z}\right)$,

and the mass $m$ is either $m_{\mu}$ for the muon or $m_{e}$ for the electron. In the Dirac representation

$$
u_{ \pm}=\not\left(\begin{array}{c}
\phi_{ \pm} \\
0
\end{array}\right)
$$

with $\rho^{\mu}=\left(\rho^{0}, \boldsymbol{\rho}\right)=\left(1, i \frac{1-\gamma}{Z \alpha} \hat{r}\right)$. Since this approach differs from Ref. [4], we present it in some detail.

\section{A. Factorizing neutrinos}

It is convenient to decompose the decay into two stages: first the muon decays into the electron and a fictitious 
spin-one boson $A$; next, boson $A$ decays into the $\nu \bar{\nu}$ pair. The kinematically allowed range of the mass $m_{A}$ of the boson $A$, parametrized by a dimensionless variable $z$, $m_{A}=z m_{\mu}$, is $z \in\left[0, z_{\max }=\left(E_{\mu}-E_{e}\right) / m_{\mu}\right]$, where $E_{\mu, e}$ are the muon and electron energies; they should be replaced by muon and electron masses in the case of a free muon decay. The decay rate is an integral over $z$,

$$
\Gamma(\mu \rightarrow e \nu \bar{\nu})=\frac{256 \pi \Gamma_{0}}{g^{2} m_{\mu}} \int_{0}^{z_{\max }} \Gamma\left[\mu^{-} \rightarrow e^{-} A\right] z^{3} \mathrm{~d} z
$$

where $g$ is the weak coupling constant. One advantage of Eq. (21) is that it holds both for a free and for a bound muon decay. It is simpler to deal with a two-body decay $\mu \rightarrow e A$ than with $\mu \rightarrow e \nu \bar{\nu}$. Binding effects as well as radiative corrections (ignored in the present paper) affect only $\Gamma(\mu \rightarrow e A)$.

As an example of using (21) consider a free muon decay. Then $z_{\max }=1-\delta, \delta=m_{e} / m_{\mu}$, and

$\Gamma(\mu \rightarrow e A)=\frac{g^{2}}{32 \pi}\left(1+z^{2}-2 z^{4}+\delta^{2} z^{2}-2 \delta^{2}+\delta^{4}\right) \frac{q(z)}{z^{2}}$,

where $q(z)$ is the momentum of $A$; for a free muon decay, $q(z)=\frac{\lambda^{1 / 2}\left(1, \delta^{2}, z^{2}\right)}{2} m_{\mu}$ with the Källén function $\lambda(x, y, z)=$ $x^{2}+y^{2}+z^{2}-2(x y+y z+z x)$. Integration over $z$ gives

$\Gamma(\mu \rightarrow e \nu \bar{\nu})=\Gamma_{0}\left(1-8 \delta^{2}-24 \delta^{4} \ln \delta+8 \delta^{6}-\delta^{8}\right)$,

reproducing the correct electron mass dependence [25].

\section{B. Decay rate}

The amplitude for the $(Z \mu) \rightarrow(Z e)+A$ transition is

$$
\mathcal{M}=\frac{g}{\sqrt{2}} \int \mathrm{d}^{3} \boldsymbol{r} \exp (i \boldsymbol{q} \cdot \boldsymbol{r}) \bar{\Phi}_{\mu}(\boldsymbol{r}) \phi^{\lambda_{A} \star} L \Phi_{e}(\boldsymbol{r}),
$$

where $L=\frac{1-\gamma_{5}}{2}$ and $\lambda_{A}$ labels the polarization state of $A$. The triple $\boldsymbol{r}$ integration is done analytically. Angular integrations lead to spherical Bessel functions, and the $r$-integration results in a relatively compact formula. After squaring the amplitude, we find, using $k_{A}=\sqrt{z_{\max }^{2}-z^{2}}$ and $a=\frac{1-\gamma}{\alpha_{Z}}$,

$$
\begin{aligned}
& \Gamma\left[\left(Z \mu^{-}\right) \rightarrow\left(Z e^{-}\right)+A\right] \\
& =\frac{m_{\mu} g^{2}}{2 \pi} k_{A}\left(N_{a}^{2}+N_{b}^{2}+F_{a}^{2}+F_{b}^{2}\right), \\
N_{a} & =\sqrt{2} \frac{z_{\max }}{z}\left[4 a^{2}\left(C_{2}-S_{3}\right)+\left(1+a^{2}\right) S_{1}\right], \\
N_{b} & =\sqrt{2} \frac{k_{A}}{z}\left(1+a^{2}\right) S_{1}, \\
F_{a} & =4 a^{2}\left(C_{2}-S_{3}\right)-2\left(1-a^{2}\right) S_{1}, \\
F_{b} & =4 a\left(S_{2}-C_{1}\right),
\end{aligned}
$$

where the quantities $C_{n}$ (and analogously $S_{n}$ with $\cos \rightarrow$ sin) are

$$
\begin{gathered}
C_{n}=\frac{1+\gamma}{8}\left(\frac{4 \delta}{(1+\delta)^{2}}\right)^{\gamma+\frac{1}{2}} \frac{\Gamma(1+2 \gamma-n)}{k^{n} \Gamma(1+2 \gamma)} \\
\times\left(1+k^{2}\right)^{\frac{n-1}{2}-\gamma} \cos [(1+2 \gamma-n) \arctan k], \\
k=\frac{k_{A}}{\alpha_{Z}(1+\delta)} .
\end{gathered}
$$

The rate $\Gamma\left[\left(Z \mu^{-}\right) \rightarrow\left(Z e^{-}\right)+\nu \bar{\nu}\right]$ can now be expressed as a single integral over $z$, a variable equivalent to the invariant mass of the neutrinos, from zero to $z_{\max }=\gamma(1-\delta)$,

$$
\begin{aligned}
& \frac{1}{\Gamma_{0}} \Gamma\left[\left(Z \mu^{-}\right) \rightarrow\left(Z e^{-}\right)+\nu \bar{\nu}\right] \\
& \quad=128 \int_{0}^{z_{\max }}\left(N_{a}^{2}+N_{b}^{2}+F_{a}^{2}+F_{b}^{2}\right) k_{A} z^{3} \mathrm{~d} z .
\end{aligned}
$$

This is the main result of this paper. Note that the position space calculation results in a single integral representation for the rate. This is much simpler than the result of Ref. [4], where two additional integrations remain, over the magnitude and the polar angle of the argument of the momentum space wave function. Those integrations seem to be more involved than the corresponding radial and angular integrations in the position space. In the following section we perform the remaining integration over $z$ in the limit of close electron and muon masses.

\section{LIMITING CASE OF SIMILAR ELECTRON AND MUON MASSES}

We expect out results to agree with Ref. [4] for small $Z$, since the only conceptual difference between our approaches involves negative energy components, and those are suppressed by powers of $\alpha_{Z}$. Here we demonstrate this agreement with a simple closed formula in the limiting case of nearly equal masses. We write

$$
m_{\mu}-m_{e}=\epsilon m_{\mu}
$$


and consider a hypothetical situation where $\epsilon$ is small. In the limit $\epsilon \rightarrow 0$, the decay rate computed in Ref. [4] [cf. Eq. (A7) in the Appendix] is

$$
\begin{aligned}
& \Gamma\left[\left(Z \mu^{-}\right) \rightarrow\left(Z e^{-}\right) \nu_{\mu} \bar{\nu}_{e}\right] \\
& \quad=\int_{0}^{m_{1}-m_{2}} d|\boldsymbol{q}| \frac{G_{F}^{2} \boldsymbol{q}^{2}}{12 \pi^{3}} K(|\boldsymbol{q}|) \\
& \quad=\gamma^{5} \epsilon^{5} \frac{G_{F}^{2} m_{\mu}^{5}}{15 \pi^{3}}\left\{\left[F_{1}(0)-F_{2}(0)\right]^{2}+F_{1}(0) F_{2}(0)\right\} .
\end{aligned}
$$

In this approximation of almost equal muon and electron masses, the momentum transferred to the neutrinos $\mathbf{q}$ is approximately zero. Therefore, the form factors $F_{1}$ and $F_{2}$ given in Eq. (30) are evaluated at $|q|=0$ which gives

$$
\begin{aligned}
& F_{1}(0)=\int \frac{d^{3} k}{(2 \pi)^{3}} \psi_{\mu}(\boldsymbol{k}) \psi_{e}^{*}(\boldsymbol{k}) \frac{2 k^{0}+m_{\mu}}{3 k^{0}}=\frac{2}{3}+\frac{1}{3}\left\langle L^{-1}\right\rangle, \\
& F_{2}(0)=\int \frac{d^{3} k}{(2 \pi)^{3}} \psi_{\mu}(\boldsymbol{k}) \psi_{e}^{*}(\boldsymbol{k}) \frac{k^{0}-m_{\mu}}{3 k^{0}}=\frac{1}{3}-\frac{1}{3}\left\langle L^{-1}\right\rangle,
\end{aligned}
$$

where the mean inverse Lorentz factor $\left\langle L^{-1}\right\rangle$ is

$$
\left\langle L^{-1}\right\rangle=\int \frac{d^{3} k}{(2 \pi)^{3}}\left|\psi_{\mu}(\boldsymbol{k})\right|^{2} \frac{m_{\mu}}{k_{1}^{0}} .
$$

Hence, Eq. (30) becomes

$$
\frac{\Gamma}{\Gamma_{0}}=\frac{64}{5} \epsilon^{5} \gamma^{5} \frac{1+\left\langle L^{-1}\right\rangle+\left\langle L^{-1}\right\rangle^{2}}{3} .
$$

Numerical results $\frac{\Gamma\left[\left(Z \mu^{-}\right) \rightarrow\left(Z e^{-}\right) \nu_{\mu} \bar{\nu}_{e}\right]}{\Gamma_{0}}$ for the limiting case of almost equal masses are shown in Table I.

In the equal mass limit, using $|\boldsymbol{q}| r \rightarrow 0$, our momentum space as well as position space treatments lead to the expression

$$
\frac{\Gamma\left[\left(Z \mu^{-}\right) \rightarrow\left(Z e^{-}\right) \nu_{\mu} \bar{\nu}_{e}\right]}{\Gamma_{0}}=\frac{64}{5} \epsilon^{5} \gamma^{5} \frac{1+\gamma+\gamma^{2}}{3} .
$$

TABLE I. Numerical values of $\Gamma\left[\left(Z \mu^{-}\right) \rightarrow\left(Z e^{-}\right) \nu_{\mu} \bar{\nu}_{e}\right] / \Gamma_{0}$ in an artificial situation with the electron only slightly lighter that the muon, $\epsilon=1-m_{e} / m_{\mu}=0.01$, for a small $Z=10$ and a large $Z=80$ : using the formalism of Ref. [4], Eq. (A6) (second column), its limit for $m_{e} \simeq m_{\mu}$, Eq. (32) (third column), and the $m_{e} \simeq m_{\mu}$ limit of our approach, Eq. (33) (fourth column). As expected, the agreement is better for small $Z$ (first line).

\begin{tabular}{lccc}
\hline \hline$Z$ & Eq. (A6) & Eq. (32) & Eq. (33) \\
\hline 10 & $1.25 \times 10^{-9}$ & $1.26 \times 10^{-9}$ & $1.26 \times 10^{-9}$ \\
80 & $3.85 \times 10^{-10}$ & $3.83 \times 10^{-10}$ & $3.72 \times 10^{-10}$ \\
\hline \hline
\end{tabular}

The analogous limit of the free muon decay rate, Eq. (23), is $\frac{64}{5} \epsilon^{5}$. Thus the binding effects given by $\gamma^{5} \frac{1+\gamma+\gamma^{2}}{3}=$ $1-3 \alpha_{Z}^{2}+\mathcal{O}\left(\alpha_{Z}^{4}\right)$ in the case of the decay into a bound electron are more pronounced than in the case of the decay into a free electron, given in Eq. (1). For a free electron, the effect can be approximated by a single factor of $\gamma \simeq 1-\alpha_{Z}^{2}+\mathcal{O}\left(\alpha_{Z}^{4}\right)$

Numerical evaluation of Eq. (33) is given in the last column of Table I. For small $Z$, we have $\left\langle L^{-1}\right\rangle \approx \gamma$ and hence the corresponding results coincide in this case of equal muon and electron masses. This is not the case for large $Z$, where $\left\langle L^{-1}\right\rangle$ is larger than $\gamma$.

\section{CONCLUSION}

We have calculated the decay rate of a bound muon to bound electron using Dirac wave functions for different values of $Z$ in two formalisms. Numerical results in momentum and in position space coincide, provided that complete wave functions (both positive and negative energy components) are used. If the negative energy parts of the wave functions are neglected, as was done in Ref. [4], the results are significantly larger. For $Z=80$ the difference is about $38 \%$.

This is surprising since the probability of finding positrons in a hydrogen-like atom or ion is very small even for $Z=80$. Our tentative interpretation is that the probability of the decay into a bound electron is very suppressed and that this suppression is relatively less severe for the negative energy components. We note that the decay vertex couples positive and negative energy components without a suppression factor of $\alpha_{Z}$. Also, the decay rate involves an interference of large $A_{ \pm}$with small $B_{ \pm}$wave function terms, whereas the normalization integral involves the small $B_{ \pm}$only in second powers, thus greatly decreasing their contribution [see Eq. (14)].

In order to check this unexpected result, we developed a position space approach. It resulted in a simple onedimensional integral representation of the rate, Eq. (28). The remaining integral has been done in the limiting case of close electron and muon masses, Eq. (33), giving a closed formula valid for all $\alpha_{Z}$.

In closing, we quote from Sidney Coleman's field theory lectures [26]: Dirac's theory gives excellent results to order $(v / c)^{2}$ for the hydrogen atom, even without considering pair production and multi-particle intermediate states. This is a fluke.

\section{ACKNOWLEDGMENTS}

A.C. thanks Andrey Volotka for conversations that stimulated this project. M.J.A. and A. C. gratefully acknowledge the hospitality of the Banff International Research Station (BIRS) where parts of this work were done. This research was supported by the Natural Sciences and Engineering Research Council of Canada. 


\section{APPENDIX: BOUND MUON DECAY IN THE FORMALISM OF REF. [4]}

In this Appendix, we summarize the formalism of Ref. [4] for the transition $B_{1} \rightarrow B_{2}+X$, where $B_{1}$ and $B_{2}$ are bound states.

The invariant amplitude of $\left(Z \mu^{-}\right) \rightarrow\left(Z e^{-}\right) \nu_{\mu} \bar{\nu}_{e}$ decay is

$$
\mathcal{M}_{B_{1} \rightarrow B_{2}}=\frac{4 G_{F}}{\sqrt{2}} \sqrt{4 m_{B_{1}} m_{B_{2}}} N_{\mu} S_{s r}^{\mu}
$$

where the subscripts $B_{1}$ and $B_{2}$ represent the $\left(Z \mu^{-}\right)$and $\left(Z e^{-}\right)$states, respectively. The masses of the bound states are, with $M$ denoting the nucleus mass (note that in our approach the nucleus is treated as infinitely heavy and $M$ does not appear),

$$
\begin{array}{ll}
m_{B_{1}}=M+m_{1}, & m_{1}=m_{\mu}-E_{b, \mu}, \\
m_{B_{2}}=M+m_{2}, & m_{2}=m_{e}-E_{b, e},
\end{array}
$$

where $E_{b, \mu(e)}$ are the binding energies. In Eq. (A1), the neutrino part is given by

$$
N_{\mu}=\bar{u}\left(p_{\nu_{\mu}}\right) \gamma_{\mu} L v\left(p_{\nu_{e}}\right),
$$

and the charged current part is

$$
S_{s r}^{\mu}=\int \frac{\mathrm{d}^{3} k_{1}}{(2 \pi)^{3}} \psi_{\mu}\left(\boldsymbol{k}_{1}\right) \psi_{e}^{*}\left(\boldsymbol{k}_{1}-\boldsymbol{q}\right) \frac{\bar{u}_{s}\left(e ; \boldsymbol{k}_{1}-\boldsymbol{q}\right)}{\sqrt{2 k_{2}^{0}}} \gamma^{\mu} L \frac{u_{r}\left(\mu ; \boldsymbol{k}_{1}\right)}{\sqrt{2 k_{1}^{0}}} .
$$

Here, $k_{1}, k_{2}, p_{\nu_{e}}$ and $p_{\nu_{\mu}}$ are the 4-momenta of the muon, electron, $\nu_{e}$ and $\nu_{\mu}$, respectively, and the subscripts $r$ and $s$ are spin indices $(r, s= \pm 1 / 2)$.

The corresponding decay rate for $\left(Z \mu^{-}\right) \rightarrow\left(Z e^{-}\right) \nu_{\mu} \bar{\nu}_{e}$ can be calculated as

$$
\mathrm{d} \Gamma=\frac{1}{2 m_{B_{1}}} \mathrm{~d} \Phi\left|\mathcal{M}_{B_{1} \rightarrow B_{2}}\right|^{2} .
$$

After integration over the phase space and neglecting terms suppressed by $1 / M$, the decay rate is [4]

$$
\Gamma=\frac{G_{F}^{2}}{12 \pi^{3}} \int_{0}^{m_{1}-m_{2}} \mathrm{~d}|\boldsymbol{q}| \boldsymbol{q}^{2} K(|\boldsymbol{q}|),
$$

where

$$
\begin{aligned}
K(|\boldsymbol{q}|)= & {\left[q^{2}+2\left(m_{1}-m_{2}\right)^{2}\right]\left(F_{1}^{2}+F_{2}^{2}\right)+\frac{q^{2}}{m_{\mu}^{2}}\left[4\left(m_{1}-m_{2}\right)^{2}-q^{2}\right]\left(F_{3}^{2}+F_{4}^{2}\right) } \\
& -6 q^{2}\left[F_{1} F_{2}+\frac{q^{2}}{m_{\mu}^{2}} F_{3} F_{4}+\frac{m_{1}-m_{2}}{m_{\mu}}\left(F_{1}-F_{2}\right)\left(F_{3}-F_{4}\right)\right]
\end{aligned}
$$

and $q^{2}=q_{0}^{2}-\boldsymbol{q}^{2}=\left(m_{1}-m_{2}\right)^{2}-\boldsymbol{q}^{2}$. The form factors $F_{i}$ are defined as

$$
F_{i}\left(q^{2}\right)=\int \frac{d^{3} k_{1}}{(2 \pi)^{3}} \psi_{\mu}\left(\boldsymbol{k}_{1}\right) \psi_{e}^{*}\left(\boldsymbol{k}_{1}-\boldsymbol{q}\right) \frac{h_{i}}{\sqrt{4 k_{1}^{0} k_{2}^{0}\left(k_{1}^{0}+m_{\mu}\right)\left(k_{2}^{0}+m_{e}\right)}},
$$

with

$$
\begin{aligned}
& h_{1}=\left(k_{1}^{0}+m_{\mu}\right)\left(k_{2}^{0}+m_{e}\right)+q^{0}\left[(1-C)\left(k_{1}^{0}+m_{\mu}\right)-C\left(k_{2}^{0}+m_{e}\right)\right]+(B-C) q_{0}^{2}+A, \\
& h_{2}=(C-B) q^{2}+2 A, \\
& h_{3}=\left[(1-C)\left(k_{1}^{0}+m_{\mu}\right)+(B-C) q_{0}\right] m_{\mu}, \\
& h_{4}=\left[C\left(k_{2}^{0}+m_{e}\right)-(B-C) q_{0}\right] m_{\mu}
\end{aligned}
$$

and the expressions of $A, B$ and $C$ are given in Eq. (35) of [4]. We mention that in the expressions for $h_{1}$ and $h_{2}$ in Eq. (A9), the sign of $A$ is different than in [4] [cf. Eq. (34) there]. 
[1] H. Uberall, Decay of $\mu^{-}$mesons bound in the K shell of light nuclei, Phys. Rev. 119, 365 (1960).

[2] D. Shoukavy, COMET status and plans, EPJ Web Conf. 212, 01006 (2019).

[3] R. H. Bernstein, The Mu2e experiment, Front. Phys. 7, 1 (2019).

[4] C. Greub, D. Wyler, S. Brodsky, and C. Munger, Atomic alchemy: Weak decays of muonic and pionic atoms into other atoms, Phys. Rev. D 52, 4028 (1995).

[5] C. Porter and H. Primakoff, The effect of Bohr orbit binding on negative $\mu$-meson $\beta$-decay, Phys. Rev. 83, 849 (1951).

[6] V. Gilinsky and J. Mathews, Decay of bound muons, Phys. Rev. 120, 1450 (1960).

[7] R. Huff, Decay rate of bound muons, Ann. Phys. (N.Y.) 16, 288 (1961).

[8] P. Hänggi, R. Viollier, U. Raff, and K. Alder, Muon decay in orbit, Phys. Lett. 51B, 119 (1974).

[9] A. Czarnecki, M. Dowling, X. Garcia i Tormo, W. J. Marciano, and R. Szafron, Michel decay spectrum for a muon bound to a nucleus, Phys. Rev. D 90, 093002 (2014).

[10] R. Szafron and A. Czarnecki, Bound muon decay spectrum in the leading logarithmic accuracy, Phys. Rev. D 94, 051301 (2016).

[11] A. Czarnecki, X. Garcia i Tormo, and W. J. Marciano, Muon decay in orbit: Spectrum of high-energy electrons, Phys. Rev. D 84, 013006 (2011).

[12] G. Breit, The magnetic moment of the electron, Nature (London) 122, 649 (1928).

[13] G. Feinberg and M. Y. Chen, The $2 S(1 / 2) \rightarrow 1 S(1 / 2)+1$ photon decay of muonic atoms and parity violating neutral current interactions, Phys. Rev. D 10, 190 (1974); Erratum, Phys. Rev. D 10, 3145 (1974).

[14] A. Barut and Y. Salamin, Relativistic theory of spontaneous emission, Phys. Rev. A 37, 2284 (1988).
[15] S. G. Karshenboim, Non-relativistic calculations of the $g$ factor of a bound electron, Phys. Lett. A 266, 380 (2000).

[16] V. M. Shabaev, Generalizations of the virial relations for the Dirac equation in a central field and their applications to the Coulomb field, J. Phys. B 24, 4479 (1991).

[17] V. M. Shabaev, Virial relations for the Dirac equation and their applications to calculations of hydrogen-like atoms, Phys. Rev. D 67, 104025 (2003); Erratum, 74, 029904 (2006).

[18] A. Czarnecki, M. Dowling, J. Piclum, and R. Szafron, TwoLoop Binding Corrections to the Electron Gyromagnetic Factor, Phys. Rev. Lett. 120, 043203 (2018).

[19] A. Czarnecki, K. Melnikov, and A. Yelkhovsky, Anomalous magnetic moment of a bound electron, Phys. Rev. A 63, 012509 (2000).

[20] U. D. Jentschura, A. Czarnecki, K. Pachucki, and V. A. Yerokhin, Mass measurements and the bound-electron $\mathrm{g}$ factor, Int. J. Mass Spectrom. 251, 102 (2006).

[21] S. Sturm, F. Köhler, J. Zatorski, A. Wagner, Z. Harman, G. Werth, W. Quint, C. H. Keitel, and K. Blaum, Highprecision measurement of the atomic mass of the electron, Nature (London) 506, 467 (2014).

[22] J. Bjorken and S. Drell, Relativistic Quantum Mechanics (McGraw-Hill, New York, 1964).

[23] W. J. Marciano, Fermi constants and, "New Physics," Phys. Rev. D 60, 093006 (1999).

[24] V. B. Berestetsky, E. M. Lifshitz, and L. P. Pitaevsky, Quantum Electrodynamics (Pergamon, Oxford, 1982).

[25] A. Pak and A. Czarnecki, Mass Effects in Muon and Semileptonic $b \rightarrow c$ Decays, Phys. Rev. Lett. 100, 241807 (2008).

[26] Lectures of Sidney Coleman on Quantum Field Theory, edited by B. G.-g. Chen, D. Derbes, D. Griffiths, B. Hill, R. Sohn, and Y.-S. Ting (World Scientific, Singapore, 2018). 